\title{
The QCD Black Disk Limit in Cosmic Ray Air Showers
}

\author{
H. J. Drescher \\ Johann Wolfgang Goethe University, Postfach 1119 32, 60054 Frankfurt, Germany
}

\begin{abstract}
We discuss particle production in the high energy limit of QCD. Due to a large gluon density, the interaction reaches the black disk limit and the projectile is resolved into its partonic structure at the saturation scale. This leads to suppression of forward particle production and hereby to a faster absorption of cosmic ray air showers. This property is most suitable for the distinction of evolution scenarios for the saturation scale, e.g. fixed and running coupling BFKL, the latter of which is favored by air shower measurements.
\end{abstract}

\section{Introduction}

The biggest uncertainty in air shower simulations is certainly the hadronic interaction model, since QCD is poorly understood at these high energies and accelerator data is not available.

Higher twist corrections become increasingly important at high energies. Attempts to account for this are the implementation of an energydependent $p_{t}$ cutoff [1] for hard scattering or the resummation of enhanced pomeron diagrams in an efficient manner [2]. This way one tries to extend the applicability of hadronic interaction models up to GZK energies, $E \approx 10^{11} \mathrm{GeV}$.

Our approach [3] is to consider the black disk limit (or black body limit - BBL) at high gluon densities within the Color Glass Condensate (CGC) approach 4, where the interaction probability is close to unity. This leads to a suppression of forward particle scattering, the most important phase space region for cosmic ray air showers.

\section{Hadron nucleus scattering at very high energies}

The elastic and total scattering cross sections for quark-nucleus scattering may be written as follows [6]:

$$
\begin{aligned}
\sigma^{\mathrm{el}} & =\int d^{2} b\left[1-\exp \left(-Q_{s}^{2} / 4 \pi \Lambda^{2}\right)\right]^{2} \\
\sigma^{\mathrm{tot}} & =2 \int d^{2} b\left[1-\exp \left(-Q_{s}^{2} / 4 \pi \Lambda^{2}\right)\right] .
\end{aligned}
$$

If $Q_{s}$ is large, the cross section approaches the geometrical limit.

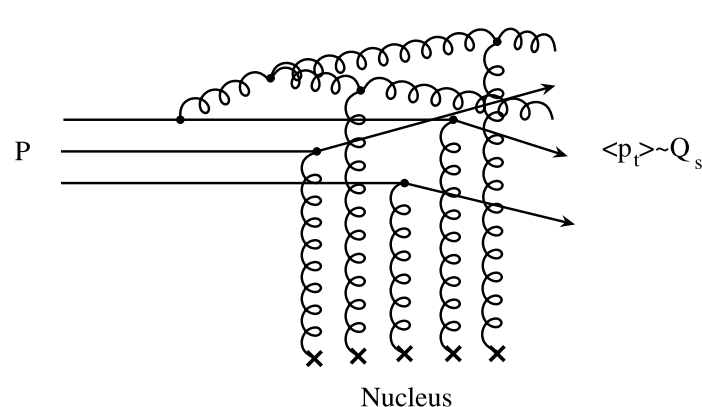

Figure 1. Schematic view of the break-up of a hadron: the partonic structure is resolved at the saturation scale $Q_{s}$

Here, two different evolution scenarios are considered for the saturation scale $Q_{S}$ : fixed coupling

$Q_{s}^{2}(x, A)=Q_{0}^{2}(A)\left(\frac{x_{0}}{x}\right)^{\lambda}$,

and running coupling $[5$

$$
\begin{aligned}
Q_{s}^{2} & =\Lambda^{2} \exp \left(\log \left(Q_{0}^{2} / \Lambda^{2}\right) \sqrt{1+2 c \alpha_{s} y}\right) \\
\alpha_{s} & =\frac{b_{0}}{\log \left(Q^{2} / \Lambda^{2}\right)}
\end{aligned}
$$

with $y=\log (1 / x)$. We assume $Q_{0}(A)$ to be proportional to the number of participants $N_{\text {part }}$. The constant $c$ assures a smooth transition to the fixed coupling scenario at low $y$. At high energies $Q_{s} \approx 5 \mathrm{GeV}$ for running coupling and $20 \mathrm{GeV}$ for fixed coupling for a central p-N collision 3 . 


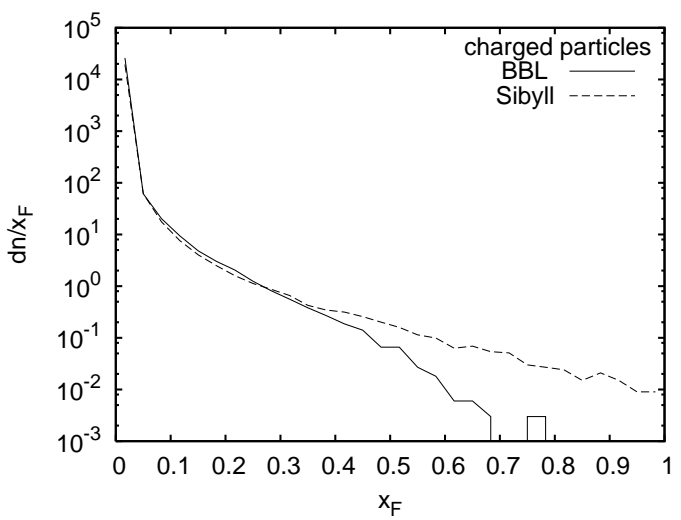

Figure 2. Suppression of forward scattering for central p-N collisions.

The diagrammatic structure of an BBL event is schematically given in Fig. 11 The projectile interacts with the target as a whole and loses its coherence [7. The partons are resolved at a scale given by the saturation momentum. An important feature is that soft physics is mostly suppressed, the typical transverse momentum being the resolution scale $Q_{s}$.

\section{Monte Carlo implementation}

Since the PDFs are resolved at the saturation scale $Q_{s}$, they provide the probability distribution for a parton to appear in the final state. The mean transverse momentum is approximately given by $Q_{s}$ :

$$
\begin{aligned}
P_{i}(x) & =f_{i}\left(Q_{s}^{2}(x), x\right) \\
\left\langle p_{t}\right\rangle & \approx Q_{s}(x)
\end{aligned}
$$

Note that $Q_{s}$ is a function of $x$ and $b$. For valence quarks we use the GRV94 parton distribution functions. For a baryon, first the three valence quarks are generated, then the remaining energy is used for gluon bremsstrahlung. Here we use the ansatz from Ref. [8]

$x g\left(x, q_{t}^{2}\right) \sim \frac{1}{\alpha_{s}} \min \left(q_{t}^{2}, Q_{s}^{2}(x)\right)(1-x)^{2}$,

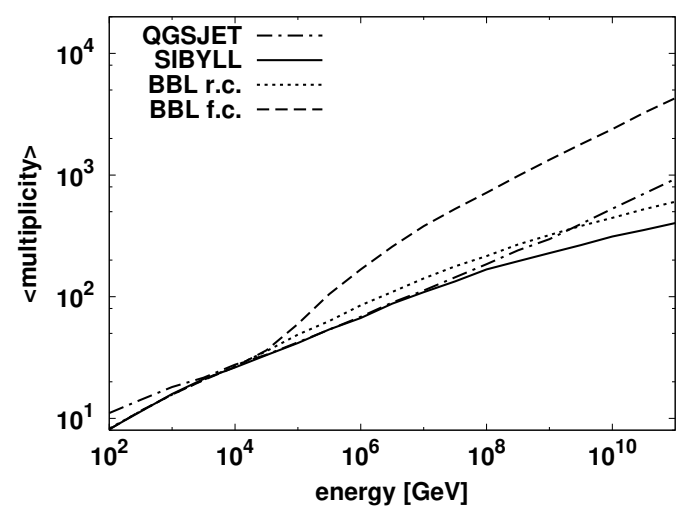

Figure 3. Mean multiplicity of central p-N events.

which exhibits no divergence for low $q_{t}$. The produced gluons are ordered in rapidity and placed on strings between the valence quarks and the target nucleus, whose precise configuration is not important, since we are interested in forward scattering. In principle, a baryon-nucleus collision produces 3 strings, a meson-nucleus collision 2 strings. However, when the invariant mass between two of the three quarks is small, one cannot assume anymore that they fragment independently. Therefore, we implement a cut-off in invariant mass,

$m_{\text {cut }}=m_{\rho}=0.77 \mathrm{GeV}$

below which two leading quarks are allowed to form a diquark. This is an important feature since it recovers the leading particle effect for low $Q_{s}$. The hadronization of the strings is done within the LUND model 9].

Of course, even at high energies, not all minimum bias events will be near the BBL. Therefore, we couple our model to a standard pQCD leading twist event generator, SIBYLL 2.1 1]. Which of the two models is to be applied for a given impact parameter, follows from the condition

$Q_{s}^{\text {Nucleus }}\left(x_{F}=0.001\right) \gtrsim 1 G e V$.

The saturation momentum of the target at a longitudinal momentum fraction of $x=0.001$ for the 


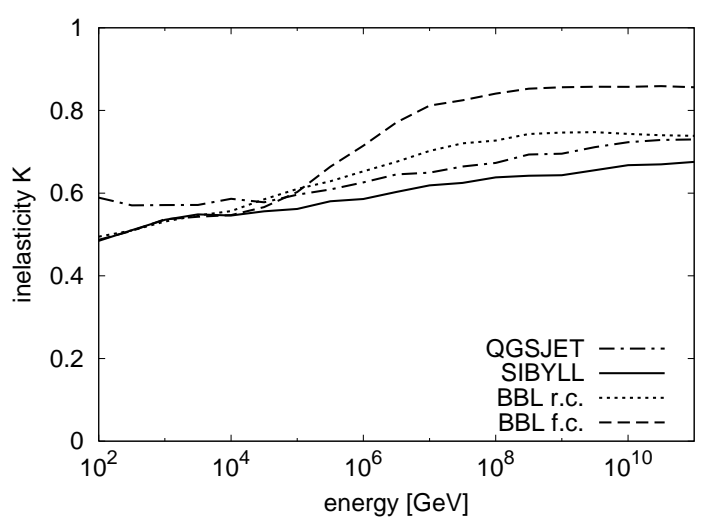

Figure 4. Inelasticity as a function of energy.

projectile must be greater than a given value. The typical resolution scale for the valence quarks is correspondingly higher, since they have $x \approx 0.2$.

\section{Results of the BBL event generator}

Using the BBL as event generator, we can compare results in particle production to the $\mathrm{pQCD}$ model SIBYLL. At the same time, we compare the two different evolution scenarios, fixed and running coupling. Fig. 22 shows a Feynman-x distribution of charged particles for central protonnitrogen collisions. One notices the absence of particle production in the very forward region, which is the typical property of this approach. This figure shows running coupling only; fixed coupling would be even more extreme. The absence of forward scattering is compensated by a relatively large multiplicity in the mid-rapidity region. In Fig. 3 we show the average multiplicities for fixed and running coupling BBL, as well as SIBYLL and QGSJET01 11] results. Due to a missing ad-hoc $p_{t}$ cutoff, QGSJET01 exhibits also a large multiplicity.

An interesting observable for air showers is the inelasticity,

$K=1-\frac{E_{\max }}{E_{0}}$,

where $E_{\max }$ is the energy of the most energetic

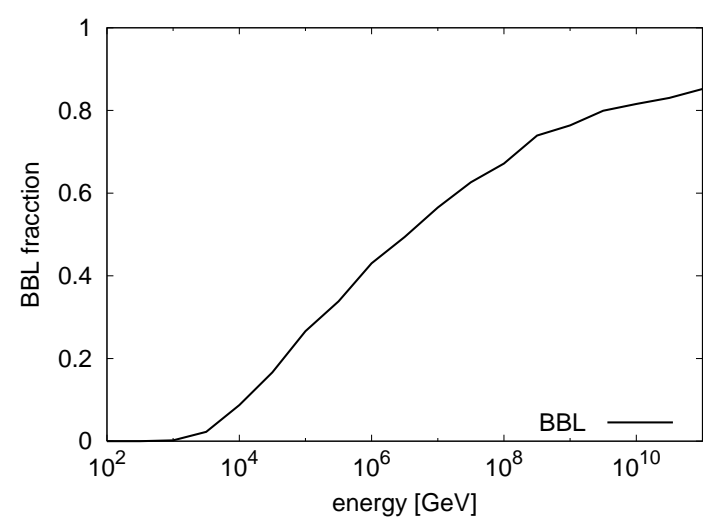

Figure 5. The fraction of BBL-events as a function of energy.

particle. A large value $K \approx 1$ means that most of the energy is used for particle production. A small value $K \approx 0$ is significant for elastic or diffractive events, where most of the energy remains in the leading particle. For air showers, a high inelasticity means that the shower are absorbed more rapidly in the atmosphere, giving

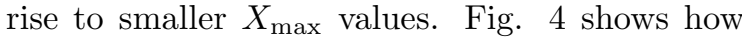
the suppression of the leading particle results in a higher $K$, especially for fixed coupling.

Finally, we show the fraction of BBL-events as a function of primary energy in Fig. 5 It reaches $90 \%$ at GZK energies. Note, that this does not mean that $90 \%$ of the cross section can be approximated as a black disk, since this also depends on the $Q_{s}$ with which the event was created. But it gives some idea about how this phenomenon becomes more important at high energies.

\section{Application of BBL to air showers}

We implement the BBL event generator into the Seneca air shower code [10 and compute the mean $X_{\max }$ as a function of energy for proton primaries.

In Figure [6] we compare the predictions of SIBYLL 2.1 for proton and iron induced showers to the saturation model (BBL, for proton primaries only) with running and fixed coupling 


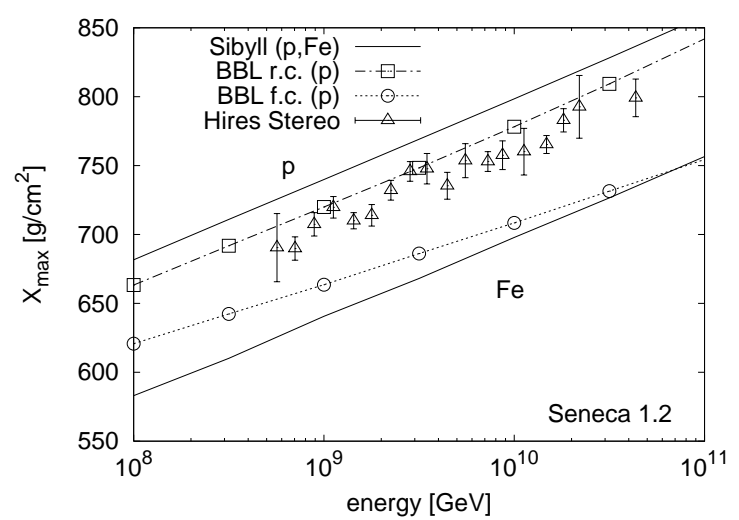

Figure 6. $X_{\max }$ as a function of primary energy.

BFKL evolution of $Q_{s}$, respectively, and to preliminary Hires stereo data [12. In the saturation limit, showers do not penetrate as deeply into the atmosphere. This is due to the "break-up" of the projectile's coherence [7] together with the suppression of forward parton scattering (for central collisions). The comparison to the data might suggest a preferably light composition, but the uncertainties at these energies are still considerable.

\section{Conclusions}

We developed an hadronic interaction model considering the black disk limit in high density QCD. The suppression of forward scattering results in faster absorption of particles within air showers, which leads to a smaller $X_{\max }$. This feature allows one to distinguish between possible evolution scenarios for the saturation momentum.

\section{Acknowledgments}

This work was done in collaboration with A. Dumitru and M. Strikman.

H.-J.D. acknowledges support by the German Minister for Education and Research (BMBF) under project DESY 05CT2RFA/7. The computations were performed at the Frankfurt Center for Scientific Computing (CSC).

\section{REFERENCES}

1. R. S. Fletcher, T. K. Gaisser, P. Lipari and T. Stanev, Phys. Rev. D 50, 5710 (1994); R. Engel, T. K. Gaisser, T. Stanev and P. Lipari, Prepared for 26th International Cosmic Ray Conference (ICRC 99), Salt Lake City, Utah, 17-25 Aug 1999.

2. S. Ostapchenko, these proceedings.

3. H. J. Drescher, A. Dumitru and M. Strikman, arXiv:hep-ph/0408073

4. L. McLerran and R. Venugopalan, Phys. Rev. D 49, 2233 (1994); ibid. 49, 3352 (1994); Y. V. Kovchegov, ibid. 54, 5463 (1996); ibid. 55, 5445 (1997).

5. E. Iancu and R. Venugopalan, arXiv:hep-ph/0303204 provides a detailed review of small- $x$ evolution and an extensive list of original references.

6. A. Dumitru and J. Jalilian-Marian, Phys. Rev. Lett. 89, 022301 (2002).

7. A. Dumitru, L. Gerland and M. Strikman, Phys. Rev. Lett. 90, 092301 (2003) [Erratum-ibid. 91, 259901 (2003)].

8. D. Kharzeev, E. Levin and L. McLerran, Phys. Lett. B 561, 93 (2003); D. Kharzeev, E. Levin and M. Nardi, Nucl. Phys. A 730, 448 (2004).

9. T. Sjöstrand, et al., Comp. Phys. Com. 135, 238 (2001).

10. G. Bossard et al., Phys. Rev. D63, 054030 (2001); H. J. Drescher and G. Farrar, Phys. Rev. D67, 116001 (2003).

11. N. N. Kalmykov, S. S. Ostapchenko and A. I. Pavlov, Nucl. Phys. Proc. Suppl. 52B, 17 (1997).

12. P. V. Sokolsky for the HiRes Collaboration, Prepared for 28th International Cosmic Ray Conference (ICRC 03), Tsukuba, Japan, July 31 - Aug. 7, 2003, p. 405; High Resolution Fly's Eye Collaboration, arXiv:astro-ph/0407622 